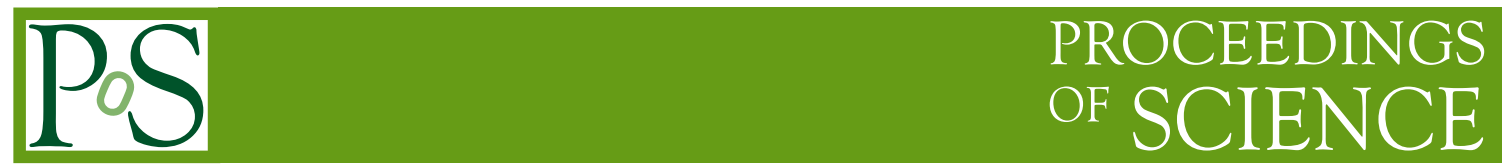

\title{
A New Experimental Method for Measuring Coherent Elastic Neutrino Nucleus Scattering (CENNS)
}

\author{
Erik Ramberg ${ }^{1}$ \\ Fermi National Accelerator Laboratory \\ PO Box 500; Batavia, Il. 60510 \\ E-mail: Ramberg@fnal.gov \\ For the \\ CENNS collaboration
}

We present a new experimental method for measuring the process of Coherent Elastic Neutrino Nucleus Scattering (CENNS). This method uses a detector situated transverse to a high energy neutrino beam production target. This detector would be sensitive to the low energy neutrinos arising from pion decays-at-rest in the target. We discuss the physics motivation for making this measurement and outline the predicted backgrounds and sensitivities using this approach. We report a measurement of neutron backgrounds as found in an off-axis surface location of the Fermilab Booster Neutrino Beam (BNB) target. The results indicate that the Fermilab BNB target is a favorable location for a CENNS experiment[1].

Technology and Instrumentation in Particle Physics 2014

2-6 June, 2014

Amsterdam, the Netherlands 


\section{Introduction}

The Coherent Elastic Neutrino-Nucleus Scattering process, or CENNS, has yet to be observed since its first theoretical prediction in 1974 by D. Freedman[2]. The condition of coherence requires sufficiently small momentum transfer to a nucleon so that the waves of offscattered nucleons in the nucleus are all in phase and add up coherently. Neutrinos with energies less than $50 \mathrm{MeV}$ largely fulfill this coherence condition in most target materials. The elastic neutral current interaction leaves no observable signature other than the low-energy recoils of the nucleus with energies of up to tens of $\mathrm{KeV}$. The technical difficulties of developing largescale, low-energy threshold, and low-background detectors have hampered the experimental realization of the CENNS measurement for more than four decades. However, recent innovations in dark matter detector technology have made the unseen CENNS testable.

Neutrinos and dark matter are similar in that they both exist ubiquitously in the Universe and interact very weakly. All major dark matter direct detection searches rely on the postulation of coherent scattering of these massive particles off of nuclei. Because of the relatively low momentum transfer, the total interaction cross-section scales as the atomic mass squared of the target material. This is an analogy for low-energy neutrinos interacting coherently with nuclei, although the mass of the incident particle is much smaller. In fact, the CENNS interactions may prove to be an irreducible background for future direct detection dark matter searches.

Besides its role as a fundamental background in dark matter searches, measurement of the CENNS process impacts a significant number of physics and astrophysics topics, including supernova explosions, neutron form factor, sterile neutrino, neutrino magnetic moments and other non-Standard Model physics.

The method we outline uses low energy neutrinos arising from stopped pion decay-at-rest source in an existing high energy neutrino beam. This differs from other methods for which detectors are proposed to be situated close to the core of a nuclear reactor[3] or spallation neutron sources[4].

In this paper, we present R\&D for a measurement of CENNS. We start by discussing the physics motivation for the CENNS process and the details of the high-intensity, low-energy neutrino flux from the Fermilab Booster Neutrino Beam (BNB). The beam-associated background and cosmogenic background measurements at the BNB target building are then described and a conceptual CENNS experiment is laid out.

\section{Physics Motivation}

\subsection{Non Standard Model Interactions}

Neutrino flavor oscillation is a well established physics phenomenon studied over the last four decades. Neutrino disappearance and appearance signatures are successfully explained by representing the neutrino flavor eigenstates as a mixture of non-zero mass eigenstates. There has been huge progress in measuring neutrino mixing angles during the last decades. Identifying mass hierarchies, measuring CP-phase(s) and determining whether neutrinos are Dirac or Majorana particles are active topics in the field. CENNS is a large and well-predicted cross- 
section in the Standard Model. If discovered at its predicted rate, the CENNS process can become a powerful tool for future low energy neutrino physics, especially for neutrino oscillation experiments.

The CENNS interaction is insensitive to the differences of active flavors of neutrinos, thus the measurement will be of total fluxes of active flavor neutrinos. Sterile neutrino oscillations manifest themselves as a baseline- and energy-dependent disappearance of CENNS interactions. A short-baseline neutrino experiment measuring CENNS has the potential to probe a wide range of oscillation hypotheses[5].

\subsection{Supernovae Neutrinos}

The major unsolved problem of a supernova explosion is to understand how the burst of neutrinos transfers its energy to produce the shock wave that causes the star to explode. CENNS plays a major role in an explosion of a core-collapse[6]. In the core of the dying star, neutrinos are scattered, absorbed, and reemitted by super-dense proton-neutron matter. Although yet to be fully understood, modern numerical simulations show that neutrino-driven convection eventually causes the giant star to explode. A CENNS cross-section different from the nominal SM prediction could have significant impact on the understanding of supernova explosions.

Moreover, CENNS is an important process for the detection of supernova neutrinos. Future large-scale low-energy threshold underground detectors, such as the CLEAN detector, will be sensitive to all active neutrino species in a supernova burst, and will be flavor blindHence, detecting supernova neutrinos in such a detector may provide a total flux and spectrum of neutrinos from supernova if the cross section of CENNS can be independently and accurately measured. These results combined with flavor-dependent interaction measurements can explain how neutrinos are thermalized with matter in a supernova[7].

\subsection{Dark Matter}

One of the most fascinating problems in Particle Astrophysics is the presence of dark matter. The Standard Model (SM) does not accommodate a suitable dark matter particle candidate; therefore dark matter is crucial phenomenological evidence for physics Beyond the Standard Model (BSM). It has been shown that the CENNS of astrophysical and atmospheric neutrinos are irreducible backgrounds for future generation dark matter detectors at spinindependent cross-sections. A recent study showed background limits to future dark matter searches coming from CENNS interactions of astrophysical and atmospheric neutrinos[8]. There are a few possible ways to improve the limits by using directional measurements of the neutrino interactions and/or measuring time variation of the interactions. However, this CENNS background limit is a robust lower bound which can not be substantially reduced. Measuring the CENNS cross section and performing subsequent tests of higher energy neutrino interactions on various target materials will be extremely beneficial to future dark matter experiments. The importance of the CENNS physics cases in dark matter searches also pointed out in a recent Snowmass report[9]. 


\section{Neutrinos from Fermilab's Booster Neutrino Beam}

Fermilab has two major neutrino beamlines: the Neutrinos at the Main Injector (NuMI) and the Booster Neutrino Beam (BNB). The energy range of these two neutrino sources on-axis is in the $\mathrm{GeV}$ range, which is too high to satisfy the condition for dominance of coherent scattering. We found the far-off-axis ( $>45$ degrees) of the BNB produces well defined neutrinos with energies below $\sim 50 \mathrm{MeV}$. The BNB source has substantial advantages over the NuMI beam source owing to suppressed kaon production from the relatively low energy $8 \mathrm{GeV}$ proton beam on the target. Therefore, pion decay and subsequent muon decay processes are the dominant sources of neutrinos. At the far-off-axis area, the detector can be placed close enough to the target to gain a large increase in neutrino flux due to the larger solid angle acceptance.

In order to understand the neutrino flux at BNB far-off-axis, we adapted the Booster Neutrino Beam Monte Carlo (BNB MC). The BNB MC uses the Geant-4 framework for propagating particles, for electromagnetic processes, hadronic interactions in the beamline materials and the decay of particles. The geometry of the target area and beamline is accurately modeled. The double differential cross sections of pion and kaon production in the simulation have been tuned to match external measurements. This is true for the hadronic cross sections for nucleons and pions as well[10]. The original BNB MC, however, contains a hard-coded tracking threshold cut to remove stopping pions (defined as below $1 \mathrm{MeV}$ in kinetic energy). In fact, the stopping pions are the dominant neutrino source at far-off-axis. The cut does not affect any previous on-axis Booster Beam experiments such as MiniBooNE and SciBooNE which focus on above-100-MeV neutrino interactions.

The existing radioactive shielding at the BNB target area is extensive and carefully planned in order to satisfy the Fermilab radioactive safety regulations .The target itself is located $\sim 7 \mathrm{~m}$ underground from the building surface. The shielding pile consists of iron blocks totaling $2.6 \mathrm{~m}$ in elevation (1,600 tons), an additional $3.2 \mathrm{~m}$-thick concrete shielding (300 tons), and special custom sized steel (40 tons) above and below the horn module. About $3 \times 10^{22}$ neutrons per $10^{21}$ protons-on-target (POT) are expected to be initially produced at the target. These neutrons are produced in the forward beam direction with a maximum kinetic energy of 8 $\mathrm{GeV}$ with more than $90 \%$ of the neutrons below $50 \mathrm{MeV}$.

The high energy neutrons scatter off the surrounding materials and produce secondaries. Considering the existing shielding configuration, the beam-induced neutron flux at about $20 \mathrm{~m}$ away from the target is roughly estimated to be $3.6 \times 10^{8}$ neutrons $/ \mathrm{m}^{2}$ per $10^{21}$ POT. According to a simple scaling of neutron shielding, an additional $8 \mathrm{~m}$-thick concrete barrier would be enough to shield out most of the beam-induced neutrons. Although the estimated beam-induced neutron background is sufficiently low, it is also true that predicting neutron leakage rates through massive shielding material is notoriously difficult. For example, a small gap between shielding blocks may potentially cause serious leakage of neutron fluxes. Fast neutron background, if there is any, would require a more extensive study for the shielding design. Therefore, measuring the beam coincident neutron flux and energy spectrum at the experimental site is necessary. With the help of the Fermilab Accelerator Division, we carried out beam-induced background studies at the BNB target building. 


\section{Measuring the Neutron Background}

A commercial EJ-301 liquid scintillator neutron de- tector and a newly-developed neutral particle detector, named SciBath[11], were used to measure the neu- tron backgrounds in the BNB target building. To obtain a rough estimate of the neutron background from the Booster beam, we attempted to measure the neutron flux with a commercial liquid scintillator detector (Eljen 510-50x50-1/301 Liquid Scintillation Detector Assembly, sealed system with 5" ETEL9390KB PMT and EJ301 scintillator). The PMT signals were recorded from $3 \mu$ s before to $20 \mu \mathrm{s}$ following the beam trigger using a CAEN V1720 $250 \mathrm{MS} / \mathrm{s}, 12-\mathrm{bit}, 2 \mathrm{Vpp}$ digitizer. The scintillation response of the cell to gammas of various energies was calibrated using the Compton edges of ${ }^{133} \mathrm{Ba},{ }^{137} \mathrm{Cs}$, and ${ }^{22} \mathrm{Na}$ sources.

Discrimination between electron recoil (gamma-induced) and nuclear recoil (neutroninduced) events can be achieved via pulse shape discrimination (PSD) [12]. We have adopted F90, the fraction of photons collected in the first $90 \mathrm{~ns}$ of a scintillation pulse, as our PSD variable. Based on its calibration, neutron events will have F90 in the range 0.76-0.91, while gammas have faster pulses with F90 $>0.91$. (Calculated values of F90 $>1$ may occur due to not accounting for baseline drift in our analysis.) Discrimination with the PSD parameter degrades rapidly at low energies due to the limited photon statistics. PSD also fails in the high energy region above the digitizer saturation point. For this reason, we restrict the neutron analysis to the region between 50 and 700 photoelectrons (p.e.), which corresponds to approximately 0.3 to 1.6 $\mathrm{MeV}$ imparted to the recoiling proton.

Figure 1 (top) shows the F90 parameter vs detection time for events in the 50-700 p.e. range. The tail of events with $\mathrm{F} 90<0.75$ is most likely due to pileup events. The $1.7 \mu$ s beam spill is evident in the region from -0.6 to $1.1 \mu$ s on this time scale, and the events in this region are overwhelmingly gamma-like; after the spill, the rate is dominated by neutron-like events. The rate of neutron-like events peaks partway through the beam spill, then decays away with a characteristic time of a few $\mu$ s. Figure 1 (bottom) shows the event energy as a function of time, from which it is clear that the energy of the neutron-like events also decays with the same few $\mu$ s timescale. Both of these observations are roughly what one would expect from neutrons gradually losing energy to elastic scattering in the shielding material and the building.

In addition to the liquid scintillator detectors, we used the SciBath detector - a prototype for the proposed FINeSSE detector which is a 13 ton, fine-grained, liquid scintillator neutrino tracking detector. While the detection concept was originally optimized to be a fine-grained neutrino tracker, it is also an excellent neutron detector. Below, we show results from a 2-month measurement of the beam-correlated neutron flux (10-200 MeV) at the BNB target building.

The SciBath detector is an 82 liter, optically-open bath of mineral-oil-based liquid scintillator that serves as both an active target and scintillator. Scintillation light is produced by the recoiling charged particles from neutral particle collisions with the mineral oil or by incoming charged particles from outside the detector. This scintillation light is absorbed by a square 16x16 array of wavelength shifting (WLS) fibers, oriented along each detector axis, with a spacing of $2.5 \mathrm{~cm}$ (i.e. 768 total fibers). The light entering each fiber is Stokes-shifted and reemitted isotropically. Some of the wavelength-shifted light is then transported by total internal reflection to a multi-anode PMT where it is read out and digitized by the DAQ. WLS fibers shift 
the ultraviolet bulk scintillation light to blue where it more effectively couples to the PMT quantum efficiency peak.

The SciBath optical properties were calibrated with cosmic ray muons and an LED pulser system. A minimum ionizing muon will deposit approximately $65 \mathrm{MeV}$ into the SciBath detector and this yields approximately 400 detected p.e. The energy deposit to light output is 6 p.e/MeV, and we found this calibration to be stable to within $5 \%$ over the entire 2 month run. A pulsed LED system was coupled to the opposite end, with respect to the PMT, of each WLS fiber. Low-light LED pulses were used to measure the single p.e. response of the PMTs and calibrate the SciBath DAQ. These LED calibrations were performed every three weeks, and the gains were stable to within $10 \%$ throughout the entire run. In fact, they were stable when compared to a previous deployment six months prior. Each PMT is mounted to a custom, Indiana-designed, "Integrated Readout Module"' (IRM) which serves as both a digitizing readout and physical mounting for the PMT. They are built on a VME form factor, but they are externally powered and connectivity is established through 1-Gigabit ethernet (in lieu of the VME power and connectivity standard). The front-end electronics of the IRM shapes and stretches the incoming pulses to enable simultaneous nanosecond timing resolution and spectroscopy with $20 \mathrm{MS} / \mathrm{s}, 12$-bit flash ADCs. Additional processing with onboard FPGAs and an ARM-9 microcontroller digitize and transfer 64 PMT channels simultaneously. For data collection, the DAQ was externally triggered on the beam for $20 \mathrm{~ms}$ with a $1 / 3$ p.e. threshold per channel and $100 \mu$ s of pre-trigger data. The LED calibration runs were also externally triggered, but only recorded $1 \mu$ s of data with no zero-suppressing threshold.

The neutron energy spectrum is unfolded from the p.e. spectrum by using the SciBath detector response as calculated with a Monte Carlo simulation. From the results of the direction spectrum, we simulated a diverging beam of neutrons with a large cross-sectional area impinging on the SciBath detector. The simulation shows that SciBath has a $0.19 \mathrm{~m}^{2}$ effective cross-sectional area for neutron acceptance. Neutrons were uniformly generated in the Monte Carlo up to $200 \mathrm{MeV}$ in $20 \mathrm{MeV}$ bins, and the simulation then tallied the total p.e. response for each $20 \mathrm{MeV}$ neutron energy bin.

The p.e. response was binned in the same as the data, 10 p.e. bins for p.e. $<1000$ and 100 p.e. bins for p.e. 1000. A least-squares fit was performed with each $20 \mathrm{MeV}$ neutron energy bin scaled by an independent fit parameter. During the fit, these fit parameters were constrained to be strictly decreasing as the neutron energy increases. These constraints were relaxed and other simulation configurations were tested in systematics tests.

The resulting neutron energy spectra changed very little as the constraints were relaxed. Figure 2 shows the unfolded neutron spectrum per pulse per $\mathrm{m}^{2}$ with the systematic uncertainties added in quadrature with the fit uncertainty. The total energy resolution is approximately $30 \%$ near the 60 p.e. threshold, and this gives an effective neutron energy threshold of approximately $10 \mathrm{MeV}$. From the unfolded neutron energy spectrum, we find the total number of neutrons above $10 \mathrm{MeV}$ per pulse per $\mathrm{m}^{2}$ is $6.3+/-0.7$. Shielding the low energy neutron flux should not be challenging, but shielding will moderate high energy neutrons to potentially dangerous energies in the CENNS detector. With this in mind, the neutron flux above $40 \mathrm{MeV}$ is particularly dangerous as a background. We measure $2.4+/-0.3$ neutrons per pulse per $\mathrm{m}^{2}$ above $40 \mathrm{MeV}$. Above $200 \mathrm{MeV}$, the SciBath detector loses sensitivity because 
recoiling protons at these energies are no longer fully contained by the detector. Fits above 200 $\mathrm{MeV}$ show very little significance, and the correlation matrix for the fit shows that we are unable to differentiate higher energy neutrons from $200 \mathrm{MeV}$ neutrons.

\section{Designing the CENNS Experiment}

Liquid Argon (LAr) has several advantages as a detection medium. As in all of the noble liquids, LAr is naturally transparent to its own scintillation light and can be made very pure, leading to long attenuation lengths for the UV photons. Most critically, the time profile of the scintillation light created by the nuclear recoil signal is dramatically different than that for electron-like backgrounds. Boulay and Hime[13] recognized that this Pulse-Shape Discrimination (PSD) allows for unprecedented rejection of ${ }^{39} \mathrm{Ar}$ beta decay background intrinsic to the argon target, a concept that has since been demonstrated in small prototype detectors and has led to major efforts for the direct detection of dark matter.

Of particular utility to a CENNS measurement is the so-called "single-phase"" approach to dark matter wherein only the primary scintillation light is recorded. This approach allows one to design a detector with the high photo-coverage necessary to achieve the desired light yield and low-energy threshold. The PMTs are the only active component in the detector, affording simplicity in design. Moreover, the speed for recording digital pulses is governed by the triplet lifetime of the argon scintillation light, thus avoiding difficulties with pulse pileup and dead time associated with a time projection chamber.

The basic conceptual design of a single-phase detector is similar to the CLEAR detector concept[3]. Key to measuring CENNS is a detector with a sufficiently large target mass and low-energy threshold to reveal a clean nuclear recoil signal that is free of background. The detector requirements for a CENNS measurement are strikingly similar to those for dark matter detection, however, with key differences: dark matter detectors need to be operated deep underground and free of cosmic ray induced background while a CENNS detector would be placed on the surface in a neutrino beam with its associated beam-related backgrounds. A great advantage of exploiting the BNB at Fermilab comes from its short-pulse time structure where $15 \mathrm{~Hz}, \mu$ s pulses provide a $5 \times 10^{-5}$ reduction factor against steady state backgrounds such as cosmic ray muons, muon-induced fast neutrons and intrinsic radioactivity in the detector itself.

The measured beam-induced neutrons (see Fig 2) can be significantly reduced with proper shieldings. The fast neutron component, above $100 \mathrm{MeV}$, requires special attention in shielding design. These neutrons may slow down in the shielding material itself and then become a more difficult background component with slower neutrons of less than a few $\mathrm{MeV}$ energy. We carried out MCNP and Geant-4 based Monte Carlo simulations in order to evaluate the overall level of neutron shielding that is needed for a CENNS experiment. We used the measured beam-induced neutron fluxes as input to the simulation. We found these neutron fluxes can be substantially suppressed by more than 7 orders of magnitude after $7 \mathrm{~m}$ of concrete shielding. We also found that measured cosmogenic neutrons can be significantly suppressed with $4 \mathrm{~m}$ of concrete shielding. Given these levels of concrete shielding, the total number of neutrons that 
enter the detector's water shielding within the detector livetime can be less than 20 neutrons $/ \mathrm{m}^{2}$ per year of operation time.

\section{Yields and Uncertainties}

There are two major sources of systematic uncertainties in a CENNS experiment: (1) Uncertainties of stopping pion production at the BNB target, and hence the related systematic uncertainties of absolute flux of neutrinos at the far-off-axis. (2) Uncertainties of scintillation yield in liquid argon detector for the measurement of low-energy nuclear recoil events. The other sources of systematics such as beam-induced neutron backgrounds, cosmogenic neutrons, gamma backgrounds, ambient radioactive decays and uncertainties from high energy neutrino interactions near or in the detector, depend on the specific experimental design or are minor background contributions.

The uncertainty in neutrino production from stopped pions and muons is dominated by the uncertainty of the pion production in the BNB target and surrounding materials. The HARP experiment at CERN measured pion production from both thin beryllium targets and a replica BNB target at the $8 \mathrm{GeV}$ proton energy that the BNB uses. The uncertainty of the pion production measured by HARP was 7\%[14]. In addition to the uncertainty in direct pion production there are uncertainties that arise from the secondary production of pions and uncertainties in the fraction of pions and muons that get to decay rather than interact. These additional uncertainties are estimated to be at the 5\% level. This gives a total of $9 \%$ neutrino flux uncertainty.

The CENNS signal is identified by single nuclear recoils in the energy range $25 \mathrm{keV}$ to $100 \mathrm{keV}$, and the most serious background is expected from nuclear recoils caused by undetected neutron scattering. An upper limit of 0.42 events $(=0.30+0.12$ events or 0.21 events after applying $50 \%$ detection efficiency) per ton-year is found for the neutrino-induced background. As the number of expected background events is small, statistical uncertainties in the simulation are not expected to be relevant. The largest systematic uncertainty of this study arises from the neutrino-argon cross-section uncertainties in the GENIE model in the relevant neutrino energy range ( $55 \mathrm{MeV}$ to $250 \mathrm{MeV}$ ), which has never been measured. However, even if we assume an order of magnitude of uncertainty in the GENIE cross section model in this energy region, the backgrounds by the high energy neutrinos are expected to be about $1 \%$ of the total number of CENNS signal events.

The neutron flux measurement by SciBath and results from a neutron shielding MC study indicate that the beam-induced neutrons can be substantially reduced with proper shielding design and could have a negligible impact on the CENNS event rates. However, due to the potential unknowns of these fast neutron shielding effects, and our current uncertainty in neutron sources and directions we assign a systematic uncertainty of beam-induced neutrons on the CENNS event rate at the $1 \%$ level.

The non-beam-related backgrounds can be significantly suppressed by the duty factor. Therefore the background requirement of the CENNS experiment is far less stringent than that of typical dark matter or other low background experiments. Cosmic-ray backgrounds can be further reduced by an active veto system in the water shielding, or it can also be significantly 
suppressed by $4 \mathrm{~m}$ of passive concrete shielding. The expected systematic uncertainty of the cosmogenic neutrons events in the signal rate is less than $1 \%$. Gamma backgrounds can also be suppressed by the duty factor, PSD and fiducial volume cuts. The backgrounds from radon daughters, especially ${ }^{210} \mathrm{Po}$ can produce nuclear recoils in the signal region. The radon daughter backgrounds in the signal region is expected to be negligible after the pulse timing cut. Moreover, the steady-state backgrounds can be separately measured by the beam-off data in the energy region of interest and can be subtracted from the signal shape. Therefore, the systematic uncertainty by radiogenic backgrounds conservatively assumed to be less than $1 \%$.

Figure 3 shows the event rate of CENNS in a one ton liquid argon neutrino detector given a neutrino flux of $5 \times 10^{5} \mathrm{v} / \mathrm{cm}^{2} / \mathrm{s}$ when the detector is located $20 \mathrm{~m}$ away from the target at a far-off-axis site. Assuming flat $\sim 50 \%$ detection efficiency, which is mostly from the PSD cut efficiency, we expect about 250 CENNS events/ton/year at $25 \mathrm{keV}$ energy threshold after background subtraction (at $32 \mathrm{~kW}$ beam power). Thus we conclude that the BNB target is a viable experimental location for a liquid Argon neutrino detector of order 1 ton in mass to make the first observation of the CENNS process at the 7 sigma level[1].

References

[1] For a comprehensive overview, see S. Brice, et. al.; Phys. Rev. D 89, 072004 (2014)

[2] Freedman, Daniel Z., Phys. Rev. D 9, 1389. (1974)

[3] P. Barbeau, J. Collar, and O. Tench, JCAP 0709, 0091245 (2007), nucl-ex/0701012

[4] K. Scholberg et al., (2009), 0910.1989

[5] G. Garvey et al., Phys.Rev. D72, 092001 (2005), hep- 1271 ph/0501013.

[6] D. Z. Freedman, D. N. Schramm, and D. L. Tubbs, 1256 Ann.Rev.Nucl.Part.Sci. 27, 167 (1977)

[7] K. Scholberg, Ann.Rev.Nucl.Part.Sci. 62, 81 (2012), 12631205.6003.

[8] J. Billard, L. Strigari, and E. Figueroa-Feliciano, (2013), 12531307.5458.

[9] P. Cushman et al., (2013), 1310.8327.

[10] A. A. Aguilar-Arevalo et al., Phys. Rev. D79, 072002 (2009), 0806.1449.

[11] R. Tayloe et al., Nucl.Instrum.Meth. A562, 198 (2006).

[12] J. M. Adams and G. White, Nucl. Instr. Meth. 156, 4951305 (1978).

[13] M. G. Boulay and A. Hime, Astropart. Phys. 25, 1791326 (2006).

[14] HARP Collaboration, M. Catanesi et al., Eur.Phys.J. C52, 29 (2007), hep-ex/0702024.
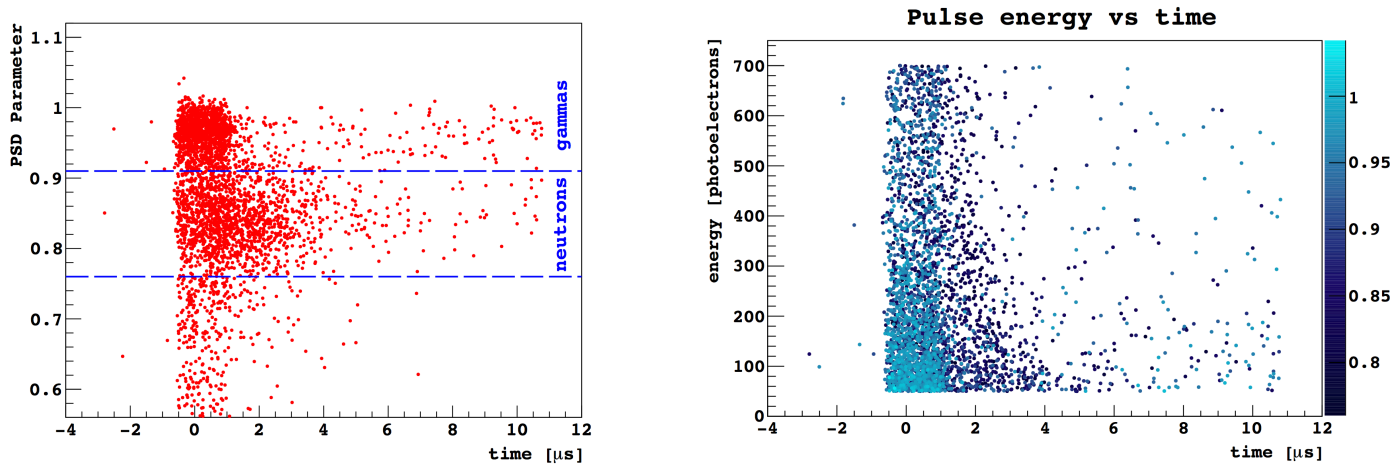

Fig. 1. Left: Pulse shape discrimination parameter of scintillation events in the EJ301 detector vs time relative to the beam trigger. Right: Energy of scintillation pulses measured in the 5" EJ301 detector vs 
time. The color scale for each point shows the PSD parameter, with darker colors being more neutronlike.

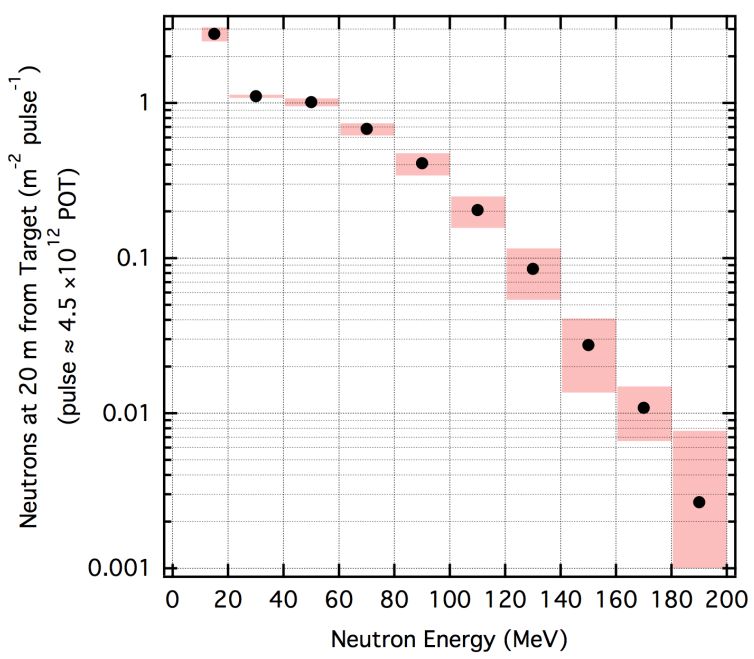

Fig. 2. The measured neutron energy spectrum by SciBath $20 \mathrm{~m}$ behind the proton target is shown. We measure $3.55+/-0.38$ neutrons per $\mathrm{m}^{2}$ per beam pulse above $40 \mathrm{MeV}$, and the low energy bin is strongly influenced by the detector threshold. The SciBath sensitivity above $200 \mathrm{MeV}$ is significantly reduced, and these energy bins have large uncertainties.

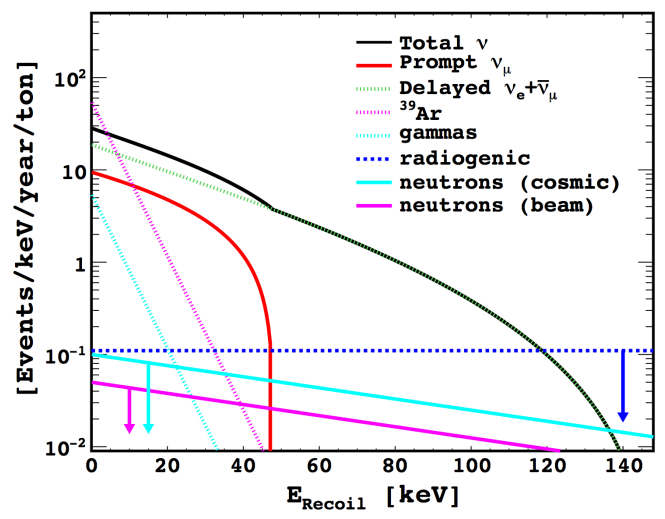

Fig. 3. Number of expected CENNS events with far-off-axis BNB (32 kW) neutrino flux. The liquid argon detector is assumed to be located at $20 \mathrm{~m}$ away from the target. The beam induced (cosmogenic) neutron background estimated based on SciBath measurements and assuming $7 \mathrm{~m}(4 \mathrm{~m})$ of concrete shielding but without water shielding. Flat $50 \%$ detection efficiencies are applied for nuclear recoil events. 